(1)

CrossMark

\title{
Airway inflammation in COPD: progress to precision medicine
}

\author{
Christopher Brightling and Neil Greening
}

Number 5 in the series

"Controversies in COPD: What Can be Done to Move the Field Forward?" Edited by D.D. Sin

Affiliation: Institute for Lung Health, NIHR Leicester Biomedical Research Centre, Dept of Respiratory Sciences, University of Leicester, Leicester, UK.

Correspondence: Christopher Brightling, Institute for Lung Health, University Hospital of Leicester, Leicester, LE3 9QP, UK. E-mail: ceb17ale.ac.uk

@ERSpublications

Airway inflammation drives COPD, but corticosteroids only work in those with eosinophilic inflammation. There is a need to better understand the patterns of inflammation, the reason for its persistence and the opportunities for new treatments. http://bit.ly/2VIOo9w

Cite this article as: Brightling C, Greening N. Airway inflammation in COPD: progress to precision medicine. Eur Respir J 2019; 54: 1900651 [https://doi.org/10.1183/13993003.00651-2019].

ABSTRACT Chronic obstructive pulmonary disease (COPD) is a significant cause of morbidity and mortality worldwide, and its prevalence is increasing. Airway inflammation is a consistent feature of COPD and is implicated in the pathogenesis and progression of COPD, but anti-inflammatory therapy is not first-line treatment. The inflammation has many guises and phenotyping this heterogeneity has revealed different patterns. Neutrophil-associated COPD with activation of the inflammasome, T1 and T17 immunity is the most common phenotype with eosinophil-associated T2-mediated immunity in a minority and autoimmunity observed in more severe disease. Biomarkers have enabled targeted antiinflammatory strategies and revealed that corticosteroids are most effective in those with evidence of eosinophilic inflammation, whereas, in contrast to severe asthma, response to anti-interleukin-5 biologicals in COPD has been disappointing, with smaller benefits for the same intensity of eosinophilic inflammation questioning its role in COPD. Biological therapies beyond T2-mediated inflammation have not demonstrated benefit and in some cases increased risk of infection, suggesting that neutrophilic inflammation and inflammasome activation might be largely driven by bacterial colonisation and dysbiosis. Herein we describe current and future biomarker approaches to assess inflammation in COPD and how this might reveal tractable approaches to precision medicine and unmask important host-environment interactions leading to airway inflammation.

Previous articles in this series: No. 1: Kim V, Aaron SD. What is a COPD exacerbation? Current definitions, pitfalls, challenges and opportunities for improvement. Eur Respir J 2018; 52: 1801261. No. 2: Washko GR, Parraga G. COPD biomarkers and phenotypes: opportunities for better outcomes with precision imaging. Eur Respir J 2018; 52: 1801570. No. 3: Soriano JB, Polverino F, Cosio BG. What is early COPD and why is it important? Eur Respir J 2018; 52: 1801448. No. 4: Leung JM, Obeidat M, Sadatsafavi M, et al. Introduction to precision medicine in COPD. Eur Respir J 2019; 53: 1802460 .

Received: March 312019 | Accepted after revision: April 252019

Copyright $\odot$ ERS 2019 


\section{Introduction}

Chronic obstructive pulmonary disease (COPD) is a common disease of chronic lung inflammation that results in persistent symptoms and fixed airflow obstruction [1]. This is caused by an inflammatory response following inhalation of cigarette smoke or other noxious external particles such as air pollution and biomass fuel [1]. Airway and systemic inflammation in COPD is related to disease progression and mortality $[1,2]$. Current diagnostic criteria do not capture the heterogeneity of COPD in terms of the complex pathological changes occurring within lung, the different airway inflammatory patterns or the airway microbial ecology. Airway inflammation is a consistent feature of COPD and is present in both the large and small airways [1,3-6]. The airway inflammation can persist after smoking cessation and is probably a consequence of altered immunity [6] and changes in the airway microenvironment [8-10].

Despite the long-standing recognition that airways inflammation is a key driver of COPD progression and exacerbations, first-line treatment strategies are aimed at symptomatic treatment of bronchoconstriction in the form of bronchodilators, rather than anti-inflammatory therapy [1]. In this review we describe the heterogeneity of airway inflammation in COPD, current and future biomarker approaches to dissect this heterogeneity and redefine COPD using multidimensional phenotyping and how this might reveal tractable approaches to precision medicine and provide important insights into the host-environment interactions.

\section{Multidimensional COPD phenotyping providing insights into pathophysiology}

COPD is a consequence of complex host-environment interactions that occur over time, summarised in figure 1. Smoking and other pollutants, pathogens and allergens insult the lung promoting airway inflammation and damage in a susceptible host as a consequence of genetic predisposition and altered immunity [6, 10-12]. In turn, this leads to irreversible damage, resulting in fixed airflow obstruction and the consequent typical symptoms of COPD.

Approaches to phenotyping airway inflammation and damage in COPD

Insights into airway inflammation and damage to the airways have been derived from lung specimens obtained from surgical resection and at post mortem. Importantly, in vivo measures of airway and systemic

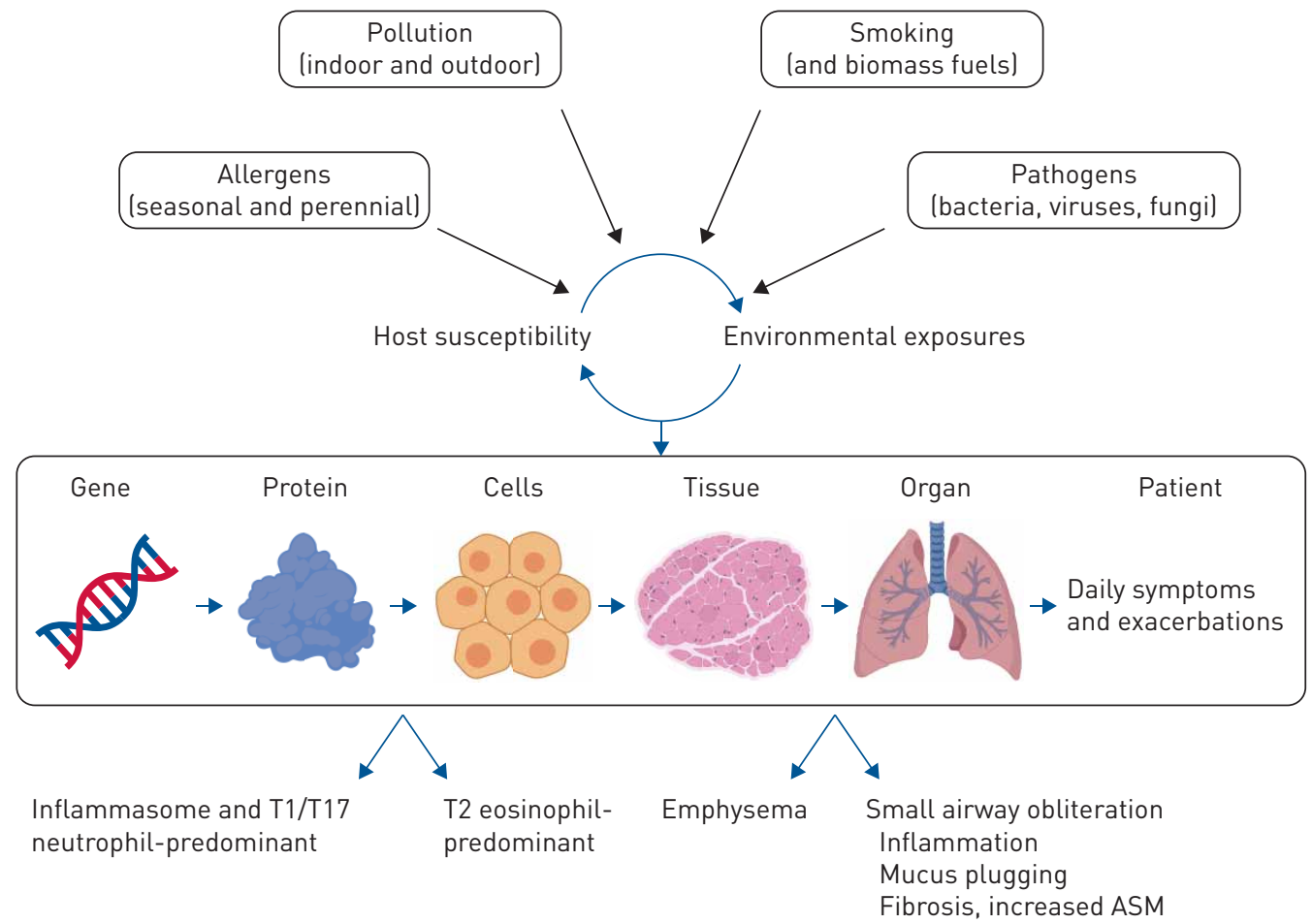

FIGURE 1 Chronic obstructive pulmonary disease is a heterogeneous complex disease resulting from complex host-environment interactions due to multiple environmental exposures over time, the host's underlying susceptibility and various host responses at the protein-to-cell and tissue-to-organ scales, leading to the clinical presentation of daily symptoms and exacerbations. ASM: airway smooth muscle. 
inflammation have been characterised longitudinally, at exacerbations and in response to therapies through invasive sampling of the airway by bronchoscopy (large airway by brush and biopsy and smaller airways by bronchoalveolar lavage); noninvasive sputum sampling (mostly large airways), which is safe even in severe COPD [13]; breath analysis (large and small airways); lung imaging (large airways directly and small airways indirectly); and beyond the lung by assessing upper airway samples and systemically using blood and urine $[5,14]$ (figure 2).

\section{Neutrophil-associated airway inflammation}

The inflammatory response in COPD involves both innate and adaptive immunity with neutrophilic inflammation the commonest inflammatory phenotype in COPD. Following exposure to cigarette smoke, other pollutants and oxidants there is airway damage [15] leading to release of pro-inflammatory mediators and damage-associated molecular patterns (DAMPs) such as interleukin (IL)-33 and thymic stromal lymphopoietin (TSLP) [15]. The distribution of the IL-33 receptor ST2 is altered in response to cigarette smoke with downregulation in innate type-2 innate lymphoid cells and upregulation by macrophages leading to a triggering of an IL-33-dependent exaggerated pro-inflammatory cascade [16]. As a consequence of airway damage the altered barrier function predisposes the airway to infection and bacterial dysbiosis, which, together with pollutants drive switching of innate lymphoid type 2 cells (ILC2) cells towards ILC1 cells, further amplifying the type-1 inflammatory cascade [17]. In COPD there is an increase in Proteobacteria and the emergence of a predominance of Haemophilus influenzae, such that the ratio of $\gamma$-Proteobacteria to Firmicutes $(\gamma \mathrm{P}: \mathrm{F})$ increases $[7-9,18]$. These pathogens themselves promote an inflammatory response via activation of pathogen-associated molecular patterns and further amplification of airway inflammation with the intensity of airway inflammation related to the abundance of $H$. influenzae $[19,20]$. In this scenario, epithelial cells are activated and are involved in the release of inflammatory mediators, such as tumour necrosis factor (TNF), IL-1 $\beta$, IL-6 and IL-8. Macrophages are recruited with further release of pro-inflammatory cytokines and activation of the NLRP3 inflammasome with caspase-1-dependent release of pro-inflammatory IL-1-like cytokines IL-1 $\alpha$, IL-1 $\beta$, IL-33 and IL-18 $[6,15]$. Activation of the inflammasome can lead to persistence of an inflammatory response by triggering an auto-inflammatory response with intrinsic production of pro-inflammatory mediators independent of exogenous stimuli [6]. Interestingly, activation of type 1 responses are more closely related to COPD

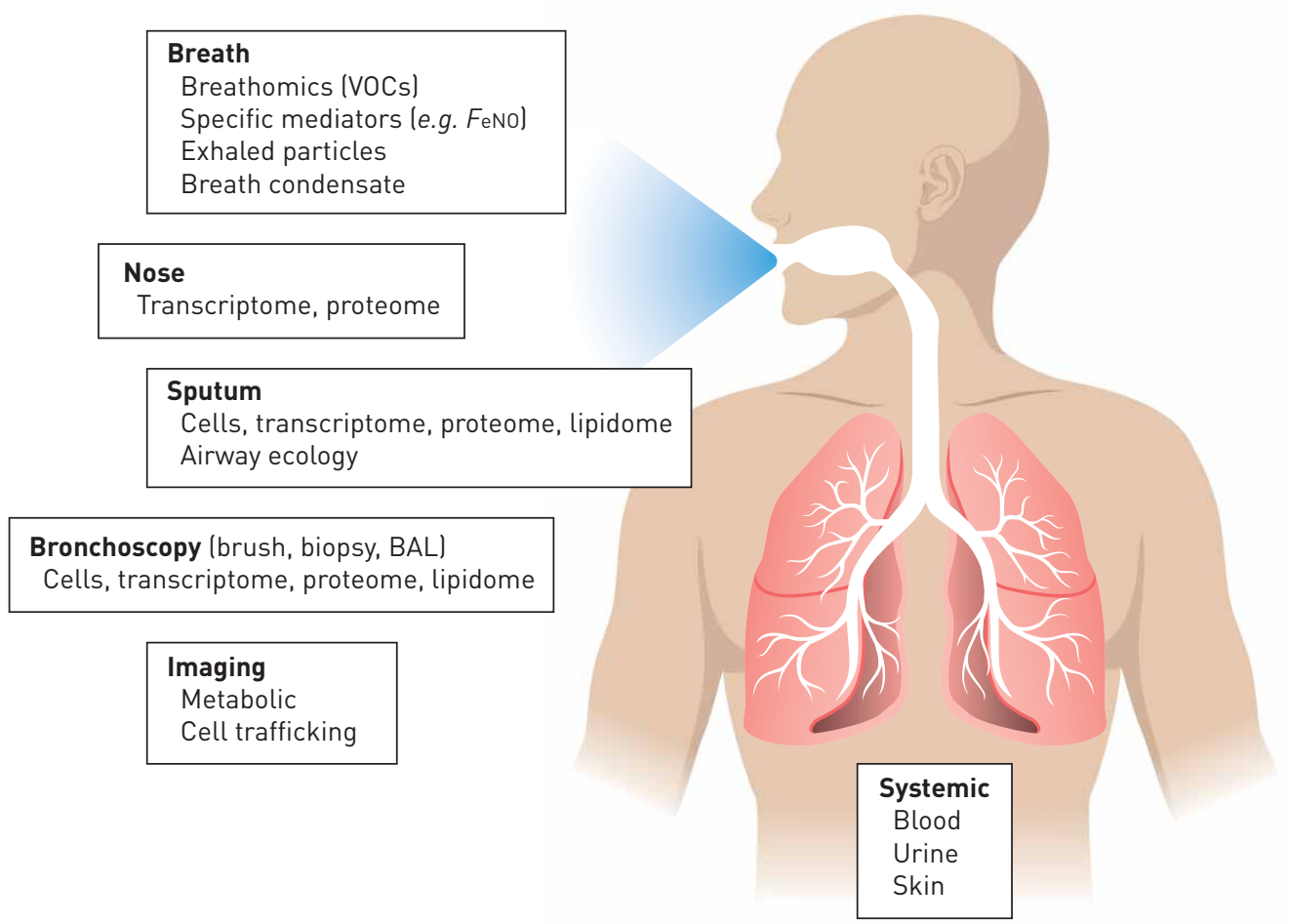

FIGURE 2 Sampling approaches to the study of inflammation in chronic obstructive pulmonary disease illustrating how these approaches in concert provide insights into the host airway and systemic inflammatory response and the local airway ecology. VOCs: volatile organic compounds; FeNo: exhaled nitric oxide fraction; BAL: bronchoalveolar lavage. 
severity than inflammasome activation, and thus autoimmunity can occur across disease severity [21]. Neutrophils are recruited as the predominant cells with consequent release of proteases and airway damage as well as activation of ILC3s. In addition, the adaptive immune response is involved with polarisation and subsequent recruitment of $\mathrm{CD}^{+}$T-helper type 1 (Th1) and 17 (Th17) cells, which produce interferon (IFN)- $\gamma$, and IL-17A and IL-17F $[6,15,22]$, respectively, with a later predominance of $\mathrm{CD}^{+} \mathrm{T}$-cells. In concert with or independent of the auto-inflammatory response there is an auto-immune response, which can also promote persistence of inflammation [6]. In more severe disease there is an accumulation of B-cells, particularly in the smaller airways, which together with T-cells and follicular dendritic cells comprise aggregates organised into tertiary lymphoid follicles [23]. These lymphoid follicles support the priming and clonal expansion of T- and B-cells with an increased proportion of IgA and B-cells, perhaps in response to increased persistent airway infection or auto-antigens [24, 25]. The cytokine network in neutrophil-associated COPD is summarised in figure $3 \mathrm{a}$.

\section{Eosinophil-associated airway inflammation}

Even though neutrophil-associated COPD is the most common inflammatory phenotype, consistent with the heterogeneity of the disease, $10-40 \%$ of COPD patients demonstrate increased eosinophilic inflammation in the sputum and or blood $[5,26,27]$ with increased T2-transcriptome signatures [28]. The broad range in prevalence is in part due to differences in patient populations, but also due to different cut-offs applied in sputum $(>2 \%$ or $>3 \%$ eosinophils $)$ or blood $(2 \%$ or $>250,>300$ or $>400$ eosinophils $\left.\mu \mathrm{L}^{-1}\right)$. Increased eosinophilic inflammation in peripheral blood and sputum samples in COPD, like asthma, is associated with a greater future risk of severe exacerbations $[29,30]$. The aetiology of eosinophilic inflammation in COPD is uncertain. As with neutrophil-associated COPD, eosinophilic COPD is likely to be a combination of innate and adaptive immunity, summarised in figure $3 \mathrm{~b}$. These pathways are well described for asthma $[5,30]$. Following allergic sensitisation and T-cell polarisation, Th2 cells produce IL-4, IL-5 and IL-13. IL-5 is an obligate cytokine for the survival and maturation of eosinophils, and IL-4 and IL-13 promote IgE production from B-cells and have direct effects upon structural cells. Recruitment of eosinophils to the lung mucosa is mediated via production of predominantly epithelium-derived CCR3 chemokines and other eosinophil chemoattractants, such as mast cell-derived prostaglandin $(\mathrm{PG}) \mathrm{D}_{2}$. $\mathrm{PGD}_{2}$ amplifies $\mathrm{T} 2$ immunity via activation of $\mathrm{PGD}_{2}$ type 2 receptors (DP2 or CRTH2). Total IgE is elevated in eosinophilic COPD, even though atopy is not increased. Whether this reflects a hitherto undescribed allergen is unclear. Eosinophilic inflammation can also occur via activation of ILC2 cells, which produce IL-5 and IL-13 in response to $\mathrm{PGD}_{2}$ and the epithelial-derived "alarmins" IL-33, IL-25 and TSLP released after epithelial damage by pollutants and microbes. Additional contributions might be from macrophage-derived IL-33, released following inflammasome activation. Whether these innate and acquired T2-mediated immune mechanisms occur in COPD, whether one is predominant over another in COPD or in asthma or whether there are alternative mechanisms driving eosinophilic inflammation in COPD remain unclear.

\section{Biological clustering to dissect heterogeneity of airways inflammation}

These eosinophilic- versus neutrophilic-associated inflammatory profiles represent extreme phenotypes. However, they are consistently reproducible and demonstrate phenotype stability $[20,26]$. In addition, the neutrophil- and eosinophil-associated phenotypes exhibit distinct microbial ecology, with $\gamma \mathrm{P}: \mathrm{F}$ predominance in the neutrophilic phenotype $[8,9,31]$. However, to describe extremes can be an oversimplification of a complex underlying biology. To validate these phenotypes and to further inform the understanding of the heterogeneity of COPD in stable state, unbiased statistical approaches such as cluster analysis have been applied to large clinical and biological datasets [18, 32, 33]. Interestingly, these have underscored the importance of eosinophilic airway inflammation in asthma, COPD and the asthmaCOPD overlap syndrome [32, 34]. Combined data from asthma and COPD revealed three biological clusters [32]. Cluster 1 consisted of asthma subjects with increased IL-5, IL-13 and CCL26 mediators and eosinophil predominance. Cluster 2 consisted of an overlap between asthma and COPD with neutrophil predominance. Cluster 3 consisted mainly of COPD patients with a mixed granulocytic airway inflammation. The differences seen between neutrophilic COPD in cluster 2 and eosinophilic COPD in cluster 3 included the presence of increased bacterial colonisation with an increased $\gamma \mathrm{P}: \mathrm{F}$ ratio in the former and increased CCL13 in the latter, possibly explaining the observed airway inflammation differences seen between these clusters (figure 4a).

Using a similar unbiased cluster analysis approach for COPD exacerbations, four biological clusters were identified and these validated the a priori aetiological groups: "pro-inflammatory" bacterial-associated, "Th1" viral-associated, "Th2" eosinophilic-associated and a fourth group that were termed "pauciinflammatory", as this was associated with limited changes in the inflammatory profile (figure 4b) [33].

Disease severity was not different between these biological clusters and the biomarkers were associated 


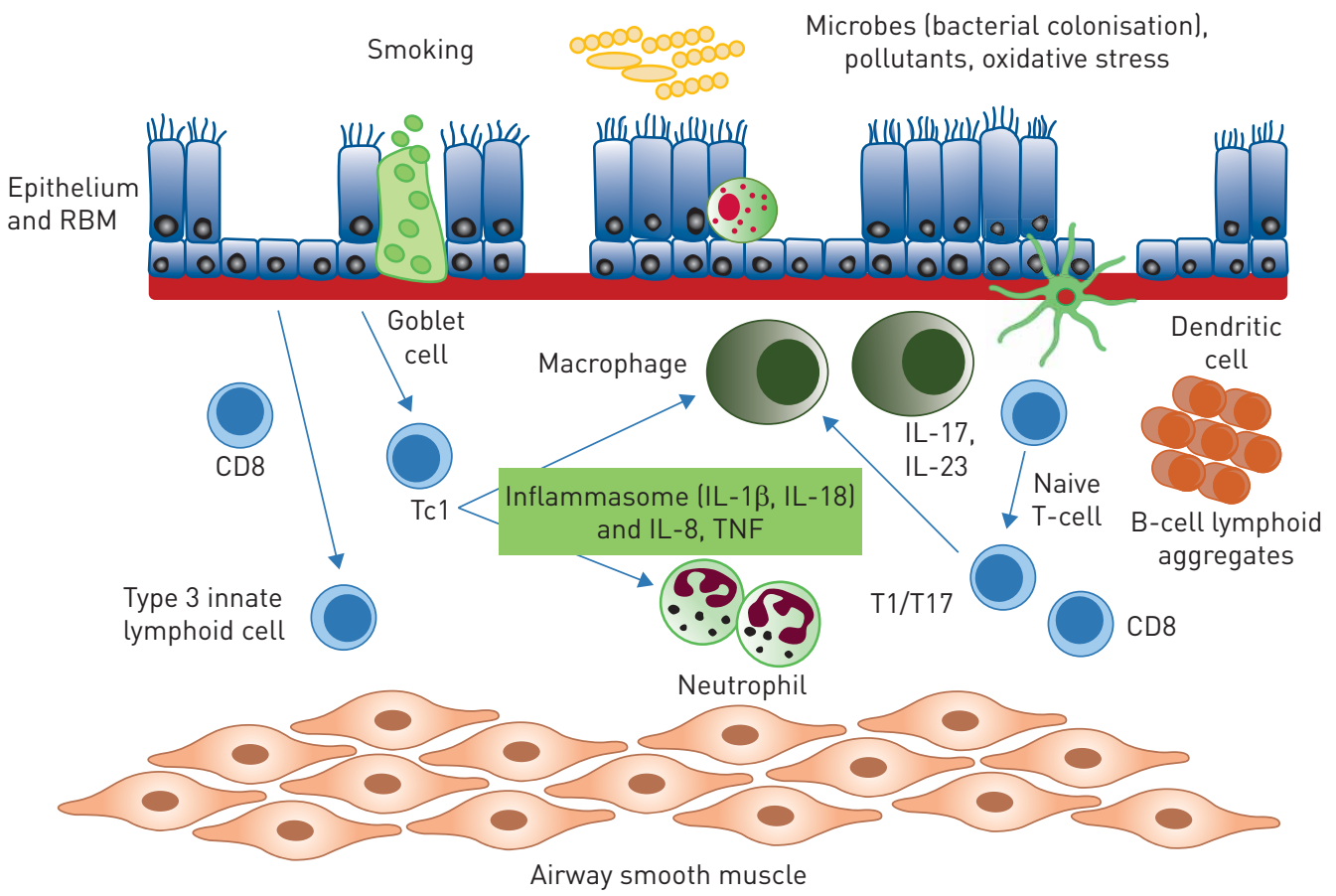

b)

\section{T2-mediated eosinophil-predominant COPD}

Pollutants, oxidative stress, microbes

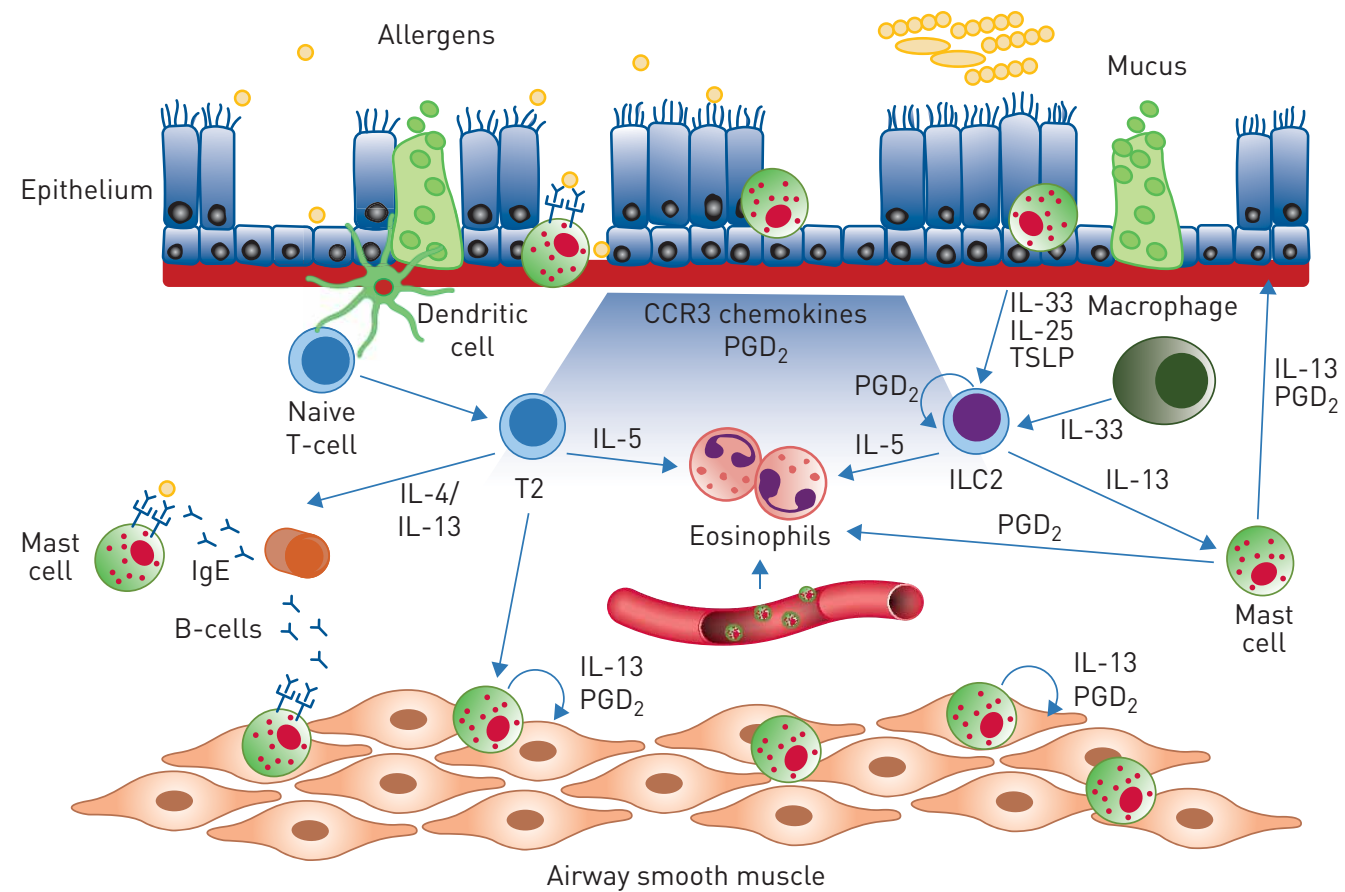

FIGURE 3 Cytokine networks in al neutrophil-associated inflammasome-mediated chronic obstructive pulmonary disease (COPD) and b) eosinophil-associated T2-mediated COPD, illustrating immunological responses to multiple environmental stimuli. RBM: reticular basement membrane; IL: interleukin; TNF: tumour necrosis factor; PGD: prostaglandin D; TSLP: thymic stromal lymphopoietin 
a)

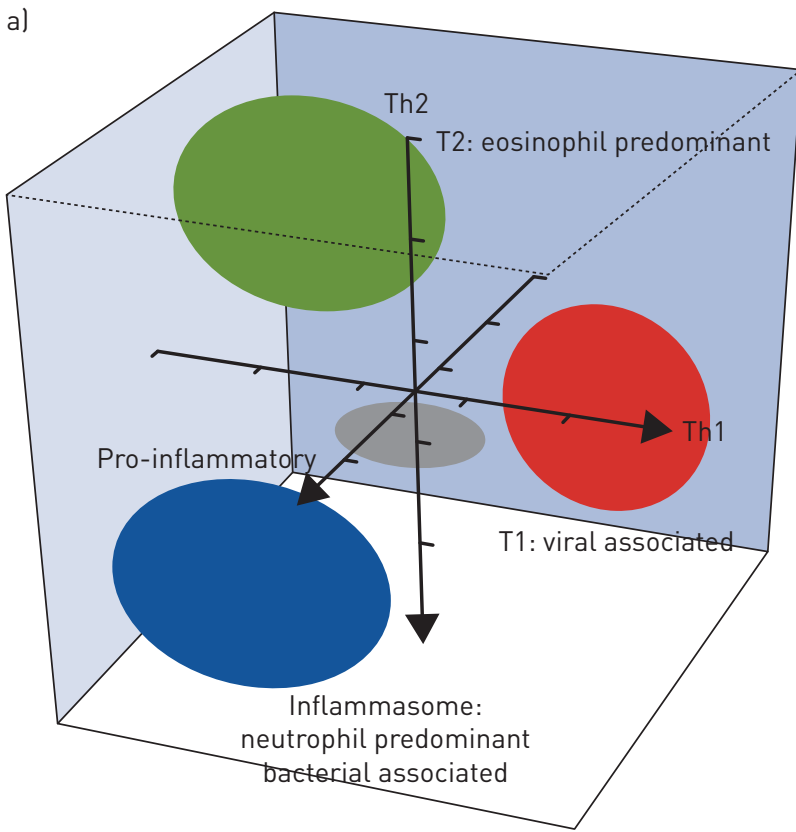

b)
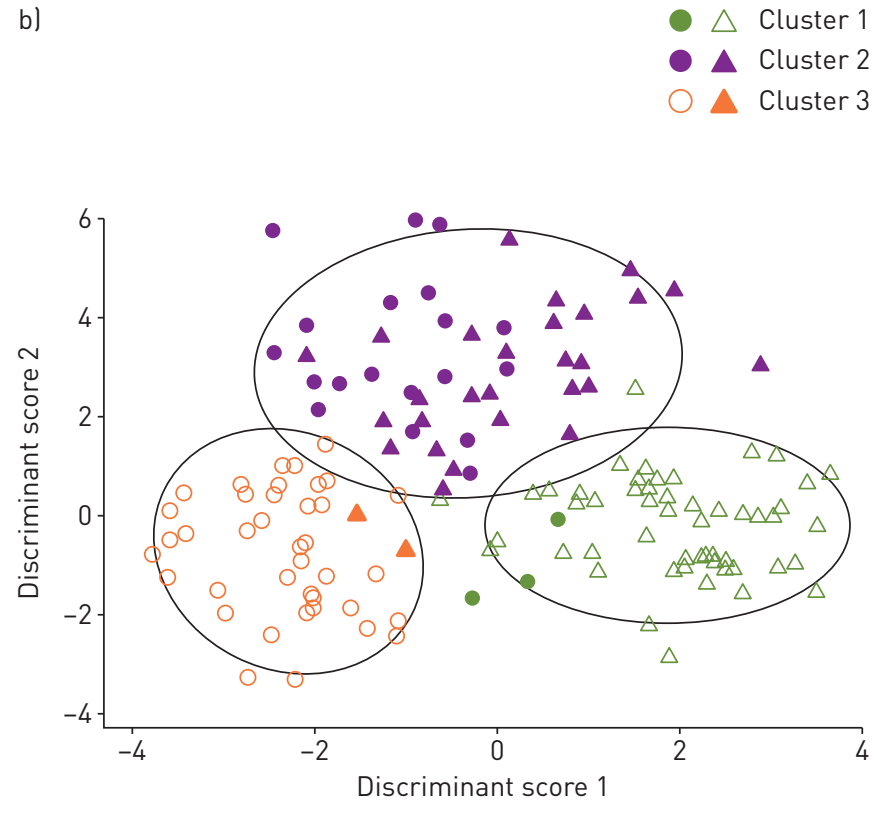

FIGURE 4 Findings from two studies. a) Biological cluster analysis of chronic obstructive pulmonary disease (COPD) exacerbations derived from multiplex of sputum mediators revealing four clusters: T2-mediated eosinophilic inflammation; T1-mediated viral associated; inflammasome-mediated bacteria-associated neutrophil-associated; and pauci-inflammatory without evidence of increased airway inflammation. Ellipsoid size is reflective of the number of patients in each cluster. b) Principal component analysis of biological clusters derived from subjects with asthma and COPD illustrating that the viral, bacterial and eosinophilic clusters are present in asthma and COPD exacerbations with different proportions represented in each cluster for each disease. The paucigranulocytic cluster was not replicated in this analysis. Cluster 1: asthma-dominant eosinophilic; cluster 2: COPD-asthma overlap neutrophilic; cluster 3: COPD-dominant mixed granulocytic. Reproduced from [32] with permission. Th1/2: T-helper type 1/2 cells.

with their respective potential aetiologies. In the pro-inflammatory bacterial-associated group the strongest discriminating inflammatory mediator was sputum IL- $1 \beta$ with increased $\gamma \mathrm{P}: \mathrm{F}$ consistent with bacterial dysbiosis. The blood eosinophil count was the best predictor of sputum eosinophilic inflammation $(>3 \%$ eosinophils) at the time of the exacerbation in this study, although the correlations are typically weaker in stable disease [35]. Interestingly, BAFADHEL et al. [33] found that patients experienced more bacterial exacerbations if their stable sputum samples contained more bacteria and high $\gamma \mathrm{P}: \mathrm{F}$ and more eosinophilic exacerbations if eosinophilic inflammation was present in the stable state, suggesting that the exacerbation event was an amplification of the underlying phenotype. Thus, in addition to directing therapy during the exacerbation event, these biomarkers might identify subgroups to target therapy in stable state with the aim of reducing future risk. The exception to this was a viral infection representing a new event and a new inflammatory profile with increased blood and sputum concentrations of the IFN-inducible chemokines CXCL10 and CXCL11.

\section{Airway damage and remodelling: emphysema and small airway obliteration}

Airway inflammation in COPD contributes to airway damage, remodelling, loss of small airways and emphysema (tissue damage with permanent dilatation distal to the terminal bronchiole). Chronic airflow obstruction is due to a combination of emphysema and small airway obliteration. Small airways are the major site of airway obstruction in COPD [48]. This small airways obliteration is due to a combination of remodelling and accumulation of inflammatory exudates within the airway lumen, both of which increase with disease severity $[36,37]$. Remodelling changes observed in COPD include disruption and loss of epithelial cilia, squamous metaplasia of the respiratory epithelium, goblet cell hyperplasia and mucous gland enlargement, bronchiolar smooth muscle hypertrophy, airway wall fibrosis and inflammatory cell infiltration [36, 37].

Computed tomography (CT) and micro CT have demonstrated a reduction in the luminal area of terminal bronchioles in COPD, but also substantial loss of terminal airways [38-40]. This is consistent with the view that the inflammation and remodelling of the small airways largely as a consequence of inflammation leads to destruction of the terminal followed by respiratory bronchioles to form centrilobular lesions. In turn, this can result in destruction of entire lung lobules which coalesce to form bullous emphysema. Thus, narrowing and consequent disappearance of small conducting airways can explain the increased peripheral airway resistance reported in COPD prior to the development of emphysema [38-40]. 
The distribution of emphysema can be centrilobular or panacinar. It is uncertain whether these represent a spectrum with panacinar a consequence of centrilobular emphysema, or if they represent distinct conditions. Panacinar emphysema is observed in individuals with $\alpha_{1}$-antitrypsin deficiency, perhaps suggesting that this form of emphysema might be largely a consequence of the imbalance between protease and anti-protease activity, whereas centrilobular emphysema is largely due to loss of and remodelling of small airways caused by persistent airway inflammation. Quantitative CT has demonstrated that small airway disease, more than emphysema is related to lung function impairment [41, 42]. These mechanisms of small airway obliteration and emphysema are important when considering anti-inflammatory therapy, as only the remaining inflamed airways can be targeted, in contrast to the airways and alveoli that are already destroyed in patients with COPD.

\section{Airway inflammation in COPD: progress to precision medicine}

Increasing knowledge of disease pathology and inflammatory phenotypes will inform our understanding of COPD and enable phenotype-specific clinical management beyond the first-line bronchodilator therapy for COPD.

\section{Eosinophilic COPD: corticosteroids}

Corticosteroids have been used in the treatment of COPD for $\geqslant 40$ years with moderate overall benefit in terms of improvement in lung function, health status, 6-min walk distance and exacerbation frequency [1]. More recently, a differential response in patients has been seen based on eosinophil count. An elevated sputum eosinophil count is associated with a greater response to both inhaled and oral corticosteroids in stable disease [43,44], while blood eosinophil count can be used to predict response to corticosteroid response in stable [45,46] and acute COPD [47], and titration of corticosteroids directed by sputum eosinophil counts reduces hospital admissions [48]. Importantly, most of these studies have recruited COPD subjects with frequent exacerbations, and thus it is uncertain whether findings can be generalised to all COPD subjects. Additionally, it is unclear whether the clinical benefits of corticosteroids, such as lung function and health status, are independent of the reduction of exacerbations. In contrast, non-T2 pathways such as IL-17 activation as determined by the epithelial IL-17A response transcriptome signature are associated with a decreased response to corticosteroids [49]. Whether the benefit from corticosteroids in COPD associated with eosinophilic inflammation is restricted to its effects upon the eosinophil or due to other broader anti-inflammatory effects is uncertain. The Global Initiative for Chronic Obstructive Lung Disease now includes the blood eosinophil count as a biomarker to direct the use of inhaled corticosteroids in COPD patients with frequent exacerbations [1]. Benefits in response to roflumilast are possibly due to attenuation of eosinophilic inflammation [50].

\section{Eosinophilic COPD: T2-targeted therapies}

Evidence for targeting T2-mediated inflammation using biologics has revolutionised clinical practice in severe asthma [30, 51]. As described earlier, significant eosinophilic inflammation does exist in COPD, albeit in a smaller proportion of patients than in asthma. However, the findings from the phase 2 and 3 trials of T2-directed therapies for COPD summarised in table 1 have been disappointing compared to asthma [52].

While a reduction in eosinophilic inflammation was observed in the first anti-IL5 receptor (R) biologic (benralizumab) trial in COPD, the primary outcome (annual rate of acute exacerbations) was not met; this included all patients with COPD, irrespective of baseline eosinophil count [53]. Importantly, the sample size was small to study exacerbations and was underpowered to observe small effects. Secondary outcomes showed an improvement in forced expiratory volume in $1 \mathrm{~s}$ in those receiving benralizumab, but no difference was observed in health status. In a pre-specified post hoc analysis, improvements in exacerbation frequency, lung function and health status were related to the intensity of baseline blood and sputum eosinophil count. In the yet to be fully reported phase 3 trials of benralizumab in COPD, the primary outcome of exacerbations in those with increased blood eosinophil count $\left(\geqslant 220\right.$ cells $\left.\mu \mathrm{L}^{-1}\right)$ was not met [54]. In a small single centre study, mepolizumab reduced sputum eosinophil count, but did not improve lung function or health status [55]. In two phase 3 trials of mepolizumab in COPD (METREX and METREO), there were small reductions in moderate or severe exacerbations in the eosinophilic subgroup $\left(\geqslant 150\right.$ cells $\left.\mu \mathrm{L}^{-1}\right)$, which was statistically significant in the METREX (18\% reduction), but not in METREO [56]. In a post hoc analysis there was no reduction in exacerbation events treated with antibiotics alone in those receiving mepolizumab versus placebo, but the reduction in exacerbations treated with oral corticosteroids with or without antibiotics was $\sim 35 \%$ in those with blood eosinophil counts $>300$ eosinophils $\mu \mathrm{L}^{-1}$. No improvements in lung function and health status in those receiving mepolizumab versus placebo were observed. 
TABLE 1 Randomised placebo-controlled trials of anti-T2 therapies in chronic obstructive pulmonary disease (COPD)

\begin{tabular}{|c|c|c|c|c|}
\hline Drug/target (study) [reference] & $\begin{array}{c}\text { Subjects } \\
n\end{array}$ & Dosage, duration & Primary outcome & Secondary outcome \\
\hline Benralizumab; anti-IL-5R [53] & 82 & $\begin{array}{c}100 \text { mg every } 4 \text { weeks ( } 3 \text { doses) } \\
\text { then every } 8 \text { weeks ( } 5 \text { doses), } \\
56 \text { weeks }\end{array}$ & $\begin{array}{l}\leftrightarrow \text { Moderate-to-severe } \\
\text { exacerbations }\end{array}$ & $\begin{array}{c}\uparrow F E V_{1} \text { in intervention } \\
\text { group } \\
\leftrightarrow \text { Health status } \\
\downarrow \text { Blood and sputum } \\
\quad \text { eosinophils }\end{array}$ \\
\hline $\begin{array}{l}\text { Benralizumab (GALATHEA); } \\
\text { anti-IL5R (NCT02138916) [54] }\end{array}$ & 1656 & $\begin{array}{c}30 \text { or } 100 \mathrm{mg} \text { every } 4 \text { weeks } \\
\text { (3 doses) then } 8 \text { weekly, } 48 \text { weeks }\end{array}$ & $\leftrightarrow$ Exacerbations & $\begin{array}{l}\downarrow \text { Blood eosinophils } \\
\leftrightarrow \text { FEV }_{1}, \mathrm{SGRQ}^{\circ}\end{array}$ \\
\hline $\begin{array}{l}\text { Mepolizumab; anti-IL-5 } \\
\text { (NCT01463644) [55] }\end{array}$ & 18 & 750 mg per month, for 6 months & $\downarrow$ Sputum eosinophils & $\begin{aligned} & \downarrow \text { Blood eosinophils } \\
\leftrightarrow & \text { FEV } 1, \text { CAT, CRQ, } \\
& \text { exacerbations }\end{aligned}$ \\
\hline $\begin{array}{l}\text { Mepolizumab; anti-IL-5 (METREO) } \\
\text { (NCT02105948) [56] }\end{array}$ & 674 & $\begin{array}{c}100 \mathrm{mg} \text { or } 300 \mathrm{mg} \text { every } 4 \text { weeks, } \\
52 \text { weeks }\end{array}$ & $\leftrightarrow$ Exacerbations & $\begin{array}{c}\leftrightarrow \text { Time to first } \\
\text { exacerbation } \\
\leftrightarrow \text { FEV1, SGRQ, CAT }\end{array}$ \\
\hline Anti-GATA3 [60] & 23 & $\begin{array}{l}\text { Inhaled } 10 \mathrm{mg} \text { SB010 twice daily, } \\
28 \text { days }\end{array}$ & Feasibility study & $\begin{array}{c}\downarrow \text { Sputum eosinophils } \\
\leftrightarrow \text { FEV }, F_{\mathrm{eNO}} \\
\text { symptoms }\end{array}$ \\
\hline
\end{tabular}

IL-R: interleukin-5 receptor; FEV1: forced expiratory volume in 1s; CAT: COPD Assessment Test; CRQ: Chronic Respiratory Disease Questionnaire; SGRQ: St George's Respiratory Questionnaire; FeNO: exhaled nitric oxide fraction; $\uparrow:$ increase; $\downarrow$ : decrease; $\leftrightarrow$ : no change.

Importantly, both the mepolizumab and benralizumab studies suggest that the effect size is smaller than that seen in severe asthma (figure 5) although, like asthma, the magnitude of benefit is directly related to the intensity of eosinophilic inflammation [57]. The subpopulation of COPD patients most likely to respond to anti-IL-5R therapy remains unclear, although it is most likely to be those with a greater disease burden and higher degree of eosinophilic inflammation. Importantly, in those with a low blood eosinophil count, there was a suggestion of a poorer outcome following treatment with anti-IL5R, which was not observed in asthma. Whether this reflects a role for the eosinophil in host defence in COPD or the importance of IL-5 in IgA B-cell differentiation [58] as a possible reason for this adverse effect in the low eosinophil group and an attenuated response in those with the same degree of eosinophilic inflammation as asthma or because the eosinophil is less important in COPD needs to be further explored. However, a small post hoc study of the effects of benralizumab upon the airway microbiome from samples obtained in

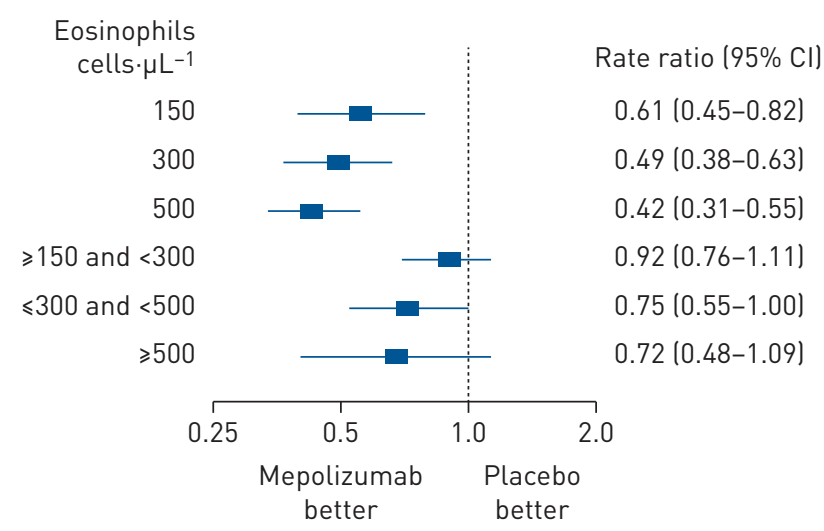

FIGURE 5 Forest plot of the effect of mepolizumab versus placebo in severe asthma derived from the MENSA trial and in chronic obstructive pulmonary disease (COPD) from the METREX and METREO trials illustrating the greater reduction in exacerbations in asthma versus COPD for the same blood eosinophil counts. 
the phase 2a study suggest that benralizumab does not have an adverse effect on the bacterial load or composition [59].

Other T2-directed therapies have been tested in COPD or are ongoing. GATA3 inhibition reduces the sputum eosinophil count in COPD, but like anti-IL5 did not affect clinical end-points [60]. A single trial of an anti-IL-13 (lebrikizumab) has been tested in COPD. The full result of the study is yet to be published, but the press release reported that COPD exacerbations were not reduced in those receiving lebrikizumab versus placebo (NCT02546700). In phase 3 studies for asthma, anti-IL-13 [51] failed to meet their primary outcome for reduction in exacerbations; in contrast, anti-IL4R $\alpha$ substantially reduced exacerbations. Whether anti-IL4R $\alpha$ has efficacy in COPD is currently being tested. The role of the DAMPs TSLP and IL-33 are also being tested in COPD. DP2 antagonism in COPD reduced the intensity of eosinophilic inflammation [61]. Whether DP2 antagonists are beneficial in a subgroup of COPD patients with underlying eosinophilic inflammation requires future studies.

Specific pro-inflammatory and pro-neutrophilic cytokines and chemokines in COPD

While the main inflammatory pathway in COPD is neutrophilic in nature, studies targeting neutrophilic inflammation have been disappointing to date (table 2). The chemokine CXCL8 (IL-8) is known to attract and activate neutrophils during an inflammatory response via the CXC chemokine receptor 1 (CXCR1) and CXCR2. In a small study a monoclonal antibody targeting IL-8 in COPD showed improved dyspnoea

TABLE 2 Randomised placebo-controlled trials of anti-neutrophil, tumour necrosis factor (TNF)- and inflammasome-targeted therapies in chronic obstructive pulmonary disease (COPD)

\section{Drug/target (study) [reference] \\ Anti-IL-8; IL-8 \\ (NCT00035828) [62]}

Anti-CXCR2 [63]

Anti-CXCR2 [64]

\author{
Infliximab; anti-TNF \\ (NCT00244192) [65] \\ Etanercept; anti-TNF
(NCT 00789997) [66] \\ Infliximab; TNF \\ (NCT00056264) [67] \\ CNTO 6785(61); \\ anti-IL-17 \\ (NCT01966549) [68]
}

22

81

186

09
$800 \mathrm{mg}$ loading dose, $400 \mathrm{mg}$ per month for 3 months, 5-month follow-up

$50 \mathrm{mg}$ twice daily or $80 \mathrm{mg}$ twice daily, 4 weeks

$10 \mathrm{mg}, 30 \mathrm{mg}$ or $50 \mathrm{mg}$, 6 months

$\downarrow$ Severity of dyspnoea as measured by TDI

Safety and tolerability

$\uparrow F V_{1}$ at 6 months

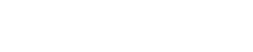

$\leftrightarrow$ Sputum inflammatory cells

$\leftrightarrow$ FEV1 over 14 days from exacerbation onset

$\leftrightarrow \mathrm{CRQ}$

$\leftrightarrow$ Pre-bronchodilator FEV1 \% predicted
$\leftrightarrow$ Moderate-to-severe exacerbations

$1 \times 1 \mathrm{mg} \cdot \mathrm{kg}^{-1}, 2 \times 3 \mathrm{mg} \cdot \mathrm{kg}^{-1}$, $42 \times 6 \mathrm{mg} \cdot \mathrm{kg}^{-1}, 45$ weeks

300 mg every 4 weeks, 52 weeks (NCT01448850) [69]

Canakinumab/IL-1 (NCT00581945) [70]

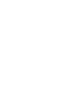

$$
\begin{gathered}
3 \mathrm{mg} \cdot \mathrm{kg}^{-1} \text { or } 5 \mathrm{mg} \cdot \mathrm{kg}^{-1} \\
44 \text { weeks }
\end{gathered}
$$

$6 \mathrm{mg} \cdot \mathrm{kg}^{-1}$ every 2 weeks for

4 weeks, then every 4 weeks for remaining 8 weeks

$5 \mathrm{mg} \cdot \mathrm{kg}^{-1}, 8$ weeks
$50 \mathrm{mg}, 90$ days
$3 \mathrm{mg} \cdot \mathrm{kg}^{-1}$ or $5 \mathrm{mg} \cdot \mathrm{kg}^{-1}$,
44 weeks
$6 \mathrm{mg} \cdot \mathrm{kg}^{-1}$ every 2 weeks for
4 weeks, then every 4 weeks for
remaining 8 weeks
$300 \mathrm{mg}$ every 4 weeks, 52 weeks
$1 \times 1 \mathrm{mg} \cdot \mathrm{kg}^{-1}, 2 \times 3 \mathrm{mg} \cdot \mathrm{kg}^{-1}$,
$42 \times 6 \mathrm{mg} \cdot \mathrm{kg}^{-1}, 45 \mathrm{weeks}$
Changes from baseline in FEV 1 , FVC

No statistical analysis provided for changes in FEV1, FVC from baseline

\section{Secondary outcome}

$\leftrightarrow$ Health status, lung function, 6MWT, rescue use of albuterol

$$
\begin{gathered}
\downarrow \text { Blood neutrophil counts } \\
\leftrightarrow \text { Time to first exacerbation } \\
\downarrow \text { Absolute and percentage } \\
\text { sputum neutrophil counts } \\
\leftrightarrow \text { SGRQ score } \\
\uparrow \text { Rate of respiratory infection } \\
\leftrightarrow \text { FEV1, SGRQ } \\
\leftrightarrow 90 \text {-day treatment failure, } \\
\text { dyspnoea, health status } \\
\leftrightarrow \text { FEV } 1,6 \text { MWT, TDI } \\
\uparrow \text { Malignancy, pneumonia } \\
\leftrightarrow \text { Post-bronchodilator FEV } 1 \% \\
\text { predicted } \\
\leftrightarrow \text { SGRQ-C } \\
\leftrightarrow \text { Frequency of AECOPD } \\
\leftrightarrow \text { Weekly usage of rescue } \\
\text { medication } \\
\leftrightarrow \text { SGRQ-C }
\end{gathered}
$$

Serious adverse events No statistical analysis provided

\footnotetext{
IL: interleukin; TDI: transition dyspnoea index; 6MWT: 6-min walk test; FEV1: forced expiratory volume in 1 s; SGRQ: St George's Respiratory Questionnaire; CRQ: Chronic Respiratory Disease Questionnaire; SGRQ-C: SGRQ for COPD patients; AECOPD: acute exacerbation of COPD; FVC: forced vital capacity; $\uparrow$ : increase; $\downarrow$ : decrease; $\leftrightarrow$ : no change.
} 
measured using the transitional dyspnoea index [62]. Anti-CXCR2 demonstrated small improvements in lung function, particularly in those who were current smokers, but did reduce exacerbations and led to increased infection rates in longer-term follow-up [63, 64]. Anti-TNF (infliximab) in COPD showed no improvements in health status, lung function, symptoms or exacerbation frequency [65-67]. Importantly, increased adverse events were noted in those receiving infliximab, including cancer and pneumonia [67]. Targeting IL-17 with biological therapy has also been ineffective in COPD [68]. The inflammasome has been targeted with two independent anti-IL-1R1 biologics $[69,70]$. In neither trial was there benefit or increased adverse events in those COPD subjects who received the biologic versus placebo.

Thus, targeting neutrophilic inflammation, the inflammasome, TNF and IL-17 have been ineffective in COPD, and in some cases have increased risk of infection. This suggests that intrinsic activation of these pathways driving an auto-inflammatory process is probably less important than their activation secondary to persistent airway colonisation and infection. It remains a possibility that targeting auto-immunity with B-cell targeted biologics could be beneficial in COPD. However, it is more likely that targeting bacterial dysbiosis in stable state and infection at exacerbation events will be more efficacious and will consequently impact upon airway inflammation. Indeed, long-term antimicrobials such as azithromycin might exert their effects largely upon the airway ecology and then ameliorate airway inflammation rather than having substantial direct anti-inflammatory effects [71, 72].

\section{Future directions}

Our current understanding of the role of different inflammatory phenotypes in COPD demonstrates that the identification of eosinophilic COPD has value in directing the use of corticosteroids in COPD. This fits with the concept of a "treatable trait" [73]. This suggests that in some COPD sufferers targeting T2-immunity beyond corticosteroids might have value. However, as described herein it is not straightforward to extrapolate findings in asthma to COPD, and the response to T2-targeted therapies is likely to be different and will need to be tested carefully for each mechanism. Notwithstanding this limitation it would seem likely that this approach will uncover further effective therapies for eosinophilic COPD patients. The impact on the airway ecology and potential risk of promoting airway infection as observed with non-T2 targeted anti-inflammatory therapies needs to be carefully studied. However, eosinophilic-associated inflammation remains a minority of patients with COPD, meaning that therapies to target other pathways are a priority. Targeting neutrophilic and inflammasome-mediated inflammation in COPD does not seem to be an attractive strategy and more attention should be focussed upon trying to normalise the airway ecology, either through novel antimicrobials or alternative strategies such as vaccines and phage therapy $[74,75]$.

Furthermore, the multidimensional phenotyping strategy suggests that the impact of the airway inflammation might have led to airway and alveoli loss, which is then not amenable to anti-inflammatory therapy. This suggests that, in contrast to asthma, the degree to which the COPD is reversible in response to anti-inflammatory therapy in established disease is limited. This will require a paradigm shift in identifying disease early and having biomarkers that are predictive of high risk of progression in order to intervene early and change the natural history of the disease. This would be similar to approaches for inflammatory joint diseases and other chronic inflammatory conditions. Genome-wide association studies have revealed multiple genes that are associated with lung function and implicated some genes involved in tissue repair and immunity. Together these genes have formed a genetic risk score for COPD. This risk score needs to be extended to identify genetic risk of disease progression or underdevelopment of full lung function with altered lung function trajectories [76] and increased likelihood of response to treatment. To date, the clinical impact of COPD genetic studies has been limited. However, the genetic risk score together with early disease biomarkers of changes in small airway disease such as oscillometry and imaging which have been extensively validated in the asthma study ATLANTIS [77] could identify at-risk groups. The longitudinal study of airway inflammation and airway ecology in these at-risk groups with "early" COPD [78] would help to define mechanism for disease onset and progression, such as whether changes in bacterial dysbiosis trigger inflammation and airway damage or a consequence of these features. Improved adoption of current biomarkers into clinical practice and the development of new simple, safe, repeatable and preferably near-patient biomarkers will provide insights of the inflammatory profile in the patient and their airway microenvironment. This will mean that the tests could be done serially to help with clinical decision-making in stable state, but also predict exacerbation events [79] prior to their onset. Breathomics is a particularly attractive approach, with early findings suggesting that this could be applied to measure airway and systemic inflammation as well as microbial dysbiosis with pathogen- and inflammatory profile-specific breath signatures beginning to be described [80]. Urine biomarkers of systemic inflammation are more distant from the lung, but could become part of clinical care with the development of home monitoring strategies for multiple inflammatory mediators coupled to artificial intelligence algorithms to provide risk stratification of future events [81]. 
Box 1 Key points

- Chronic obstructive pulmonary disease (COPD) results from an abnormal inflammatory response which is highly heterogeneous in nature

- Eosinophilic COPD is responsive to corticosteroids and identifies those most likely to respond to T2-targeted biological therapy

- Treatments to target neutrophilic inflammation have failed to show efficacy

- Neutrophilic inflammation is likely to be a consequence of changes in microbial ecology

\section{Conclusion}

In conclusion, airway inflammation is a consistent feature of COPD and is implicated in the pathogenesis and progression of COPD. Inflammation in COPD is heterogeneous, underscoring the need for a precision medicine approach (box 1) [82]. Corticosteroids are most effective in those with eosinophilic inflammation. Anti-IL-5 biologics have been disappointing in COPD versus asthma, suggesting that the role of the eosinophil is different in COPD. However, the response to corticosteroids and partial response to anti-IL-5 in this group does suggest that it is a tractable phenotype and further studies of mechanism and alternative interventions are warranted. Therapies targeting neutrophilic inflammation and the inflammasome have been ineffective and in some cases increased risk of infection, suggesting that their activation might be a consequence of bacterial colonisation and dysbiosis. Underscoring the need to focus on bacterial dysbiosis as a target to then secondarily attenuate airway inflammation. Therefore, to realise anti-inflammatory precision medicine in COPD we need to stop chasing rainbows and improve the characterisation of the disease to reflect the complexity of the multidimensional mechanisms driving COPD in individual patients.

Conflict of interest: C. Brightling reports grants and personal fees (paid to institution) for consultancy from MedImmune, AstraZeneca, GlaxoSmithKline, Roche/Genentech, Novartis, Chiesi, Pfizer and Mologic, personal fees (paid to institution) for consultancy from Teva, Sanofi, Regeneron, Glenmark and Vectura, outside the submitted work. N. Greening reports personal fees for consultancy and non-financial support for travel from AstraZeneca, Chiesi and Boehringer Ingelheim, grants, personal fees for consultancy and non-financial support for travel from GlaxoSmithKline, outside the submitted work.

\section{References}

1 Vogelmeier CF, Criner GJ, Martinez FJ, et al. Global Strategy for the Diagnosis, Management, and Prevention of Chronic Obstructive Lung Disease 2017 Report: GOLD Executive Summary. Eur Respir J 2017; 49: 1700214.

2 Celli BR, Locantore N, Yates J, et al. Inflammatory biomarkers improve clinical prediction of mortality in chronic obstructive pulmonary disease. Am J Respir Crit Care Med 2012; 185: 1065-1072.

3 Saetta M, Di Stefano A, Turato G, et al. $\mathrm{CD}^{+} \mathrm{T}$-lymphocytes in peripheral airways of smokers with chronic obstructive pulmonary disease. Am J Respir Crit Care Med 1998; 157: 822-826.

4 Di Stefano A, Capelli A, Lusuardi M, et al. Severity of airflow limitation is associated with severity of airway inflammation in smokers. Am J Respir Crit Care Med 1998; 158: 1277-1285.

5 George L, Brightling CE. Eosinophilic airway inflammation: role in asthma and chronic obstructive pulmonary disease. Ther Adv Chronic Dis 2016; 7: 34-51.

6 Scambler T, Holbrook J, Savic S, et al. Autoinflammatory disease in the lung. Immunology 2018; in press [https:// doi.org/10.1111/imm.12937].

7 Hilty M, Burke C, Pedro H, et al. Disordered microbial communities in asthmatic airways. PLoS One 2010; 5: e8578.

8 Wang Z, Singh R, Miller BE, et al. Sputum microbiome temporal variability and dysbiosis in chronic obstructive pulmonary disease exacerbations: an analysis of the COPDMAP study. Thorax 2018; 73: 331-338.

9 Wang Z, Bafadhel M, Haldar K, et al. Lung microbiome dynamics in COPD exacerbations. Eur Respir J 2016; 47: $1082-1092$.

10 Wain LV, Shrine N, Artigas MS, et al. Genome-wide association analyses for lung function and chronic obstructive pulmonary disease identify new loci and potential druggable targets. Nat Genet 2017; 49: 416-425.

11 Sakornsakolpat P, Prokopenko D, Lamontagne M, et al. Genetic landscape of chronic obstructive pulmonary disease identifies heterogeneous cell-type and phenotype associations. Nat Genet 2019; 51: 494-505.

12 Turino GM, Seniorrm, Garg BD, et al. Serum elastase inhibitor deficiency and $\alpha_{1}$-antitrypsin deficiency in patients with obstructive emphysema. Science 1969; 165: 709-711.

13 Brightling CE, Monterio W, Green RH, et al. Induced sputum and other outcome measures in chronic obstructive pulmonary disease: safety and repeatability. Respir Med 2001; 95: 999-1002.

14 Brightling CE. Chronic obstructive pulmonary disease phenotypes, biomarkers, and prognostic indicators. Allergy Asthma Proc 2016; 37: 432-438.

15 Caramori G, Casolari P, Barczyk A, et al. COPD immunopathology. Semin Immunopathol 2016; 38: 497-515.

16 Kearley J, Silver JS, Sanden C, et al. Cigarette smoke silences innate lymphoid cell function and facilitates an exacerbated type I interleukin-33-dependent response to infection. Immunity 2015; 42: 566-579.

17 Silver JS, Kearley J, Copenhaver AM, et al. Inflammatory triggers associated with exacerbations of COPD orchestrate plasticity of group 2 innate lymphoid cells in the lungs. Nat Immunol 2016; 17: 626-635.

18 Ghebre MA, Pang PH, Diver S, et al. Biological exacerbation clusters demonstrate asthma and chronic obstructive pulmonary disease overlap with distinct mediator and microbiome profiles. J Allergy Clin Immunol 2018; 141: 2027-2036. 
19 Barker BL, Haldar K, Patel H, et al. Association between pathogens detected using quantitative polymerase chain reaction with airway inflammation in COPD at stable state and exacerbations. Chest 2015; 147: 46-55.

20 Bafadhel M, Haldar K, Barker B, et al. Airway bacteria measured by quantitative polymerase chain reaction and culture in patients with stable COPD: relationship with neutrophilic airway inflammation, exacerbation frequency, and lung function. Int J Chron Obstruct Pulmon Dis 2015; 10: 1075-1083.

21 Di Stefano A, Caramori G, Barczyk A, et al. Innate immunity but not NLRP3 inflammasome activation correlates with severity of stable COPD. Thorax 2014; 69: 516-524.

22 Doe C, Bafadhel M, Siddiqui S, et al. Expression of the T helper 17-associated cytokines IL-17A and IL-17F in asthma and COPD. Chest 2010; 138: 1140-1147.

23 Hogg JC, Chu F, Utokaparch S, et al. The nature of small-airway obstruction in chronic obstructive pulmonary disease. N Engl J Med 2004; 350: 2645-2653.

24 Núñez B, Sauleda J, Antó JM, et al. Anti-tissue antibodies are related to lung function in chronic obstructive pulmonary disease. Am J Respir Crit Care Med 2011; 183: 1025-1031.

25 Ladjemi MZ, Martin C, Lecocq M, et al. Increased IgA expression in lung lymphoid follicles in severe chronic obstructive pulmonary disease. Am J Respir Crit Care Med 2019; 199: 592-602.

26 Singh D, Kolsum U, Brightling CE, et al. Eosinophilic inflammation in COPD: prevalence and clinical characteristics. Eur Respir J 2014; 44: 1697-1700.

27 Yun JH, Lamb A, Chase R, et al. Blood eosinophil count thresholds and exacerbations in patients with chronic obstructive pulmonary disease. J Allergy Clin Immunol 2018; 141: 2037-2047.

28 Christenson SA, Steiling K, van den Berge M, et al. Asthma-COPD overlap. Clinical relevance of genomic signatures of type 2 inflammation in chronic obstructive pulmonary disease. Am J Respir Crit Care Med 2015; 191: $758-766$.

29 Bafadhel M, Peterson S, De Blas MA, et al. Predictors of exacerbation risk and response to budesonide in patients with chronic obstructive pulmonary disease: a post-hoc analysis of three randomised trials. Lancet Respir Med 2018; 6: 117-126.

30 Papi A, Brightling C, Pedersen SE, et al. Asthma. Lancet 2018; 391: 783-800.

31 Kolsum U, Donaldson GC, Singh R, et al. Blood and sputum eosinophils in COPD; relationship with bacteria load. Respir Res 2017; 18: 88 .

32 Ghebre MA, Bafadhel M, Desai D, et al. Biological clustering supports both "Dutch" and "British" hypotheses of asthma and chronic obstructive pulmonary disease. J Allergy Clin Immunol 2015; 135: 63-72.

33 Bafadhel M, McKenna S, Terry S, et al. Acute exacerbations of chronic obstructive pulmonary disease: identification of biologic clusters and their biomarkers. Am J Respir Crit Care Med 2011; 184: 662-671.

34 Woodruff PG, van den Berge M, Boucher RC, et al. American Thoracic Society/National Heart, Lung, and Blood Institute Asthma-Chronic Obstructive Pulmonary Disease Overlap Workshop Report. Am J Respir Crit Care Med 2017; 196: 375-381.

35 Hastie AT, Martinez FJ, Curtis JL, et al. Association of sputum and blood eosinophil concentrations with clinical measures of COPD severity: an analysis of the SPIROMICS cohort. Lancet Respir Med 2017; 5: 956-967.

36 Bosken $\mathrm{CH}$, Wiggs BR, Paré PD, et al. Small airway dimensions in smokers with obstruction to airflow. Am Rev Respir Dis 1990; 142: 563-570.

37 McDonough JE, Yuan R, Suzuki M, et al. Small-airway obstruction and emphysema in chronic obstructive pulmonary disease. N Engl J Med 2011; 365: 1567-1575.

38 Hogg JC, McDonough JE, Suzuki M. Small airway obstruction in COPD: new insights based on micro-CT imaging and MRI imaging. Chest 2013; 143: 1436-1443.

39 Vasilescu DM, Martinez FJ, Marchetti N, et al. Non-invasive imaging biomarker identifies small airway damage in severe COPD. Am J Respir Crit Care Med 2019; in press [https://doi.org/10.1164/rccm.201811-2083OC].

40 Suzuki M, Sze MA, Campbell JD, et al. The cellular and molecular determinants of emphysematous destruction in COPD. Sci Rep 2017; 7: 9562.

41 Hartley RA, Barker BL, Newby C, et al. Relationship between lung function and quantitative computed tomographic parameters of airway remodeling, air trapping, and emphysema in patients with asthma and chronic obstructive pulmonary disease: a single-center study. J Allergy Clin Immunol 2016; 137: 1413-1422.

42 Washko GR, Parraga G. COPD biomarkers and phenotypes: opportunities for better outcomes with precision imaging. Eur Respir J 2018; 52: 1801570.

43 Brightling CE, McKenna S, Hargadon B, et al. Sputum eosinophilia and the short term response to inhaled mometasone in chronic obstructive pulmonary disease. Thorax 2005; 60: 193-198.

44 Brightling CE, Monteiro W, Ward R, et al. Sputum eosinophilia and short-term response to prednisolone in chronic obstructive pulmonary disease: a randomised controlled trial. Lancet 2000; 356: 1480-1485.

45 Steven Pascoe NL, Dransfield M, Barns N, et al. Blood eosinophil counts, exacerbations, and response to the addition of inhaled fluticasone furoate to vilanterol in patients with chronic obstructive pulmonary disease: a secondary analysis of data from two parallel randomised controlled trials. Lancet Respir Med 2015; 3: 435-442.

46 Bafadhel M, McKenna S, Terry S, et al. Blood eosinophils to direct corticosteroid treatment of exacerbations of chronic obstructive pulmonary disease: a randomized placebo-controlled trial. Am J Respir Crit Care Med 2012; 186: 48-55.

47 Bafadhel M, Davies L, Calverley PM, et al. Blood eosinophil guided prednisolone therapy for exacerbations of COPD: a further analysis. Eur Respir J 2014; 44: 789-791.

48 Siva R, Green RH, Brightling CE, et al. Eosinophilic airway inflammation and exacerbations of COPD: a andomised controlled trial. Eur Respir J 2007; 29: 906-913.

49 Christenson SA, van den Berge M, Faiz A, et al. An airway epithelial IL-17A response signature identifies a steroid-unresponsive COPD patient subgroup. J Clin Invest 2019; 129: 169-181.

50 Rabe KF, Watz H, Baraldo S, et al. Anti-inflammatory effects of roflumilast in chronic obstructive pulmonary disease (ROBERT): a 16-week, randomised, placebo-controlled trial. Lancet Respir Med 2018; 6: 827-836.

51 Diver S, Russell RJ, Brightling CE. New and emerging drug treatments for severe asthma. Clin Exp Allergy 2018; 48: 241-252.

52 Yousuf A, Brightling CE. Biologic drugs: a new target therapy in COPD? COPD 2018; 15: 99-107. 
53 Brightling CE, Bleecker ER, Jr PR, et al. Benralizumab for chronic obstructive pulmonary disease and sputum eosinophilia: a randomised, double-blind, placebo-controlled, phase 2a study. Lancet Respir Med 2014; 2: 891-901.

54 Criner GJ, Celli BR, Brightling CE, et al. Benralizumab for the prevention of COPD exacerbations. $N$ Engl J Med 2019; in press [https://doi.org/10.1056/NEJMoa1905248].

55 Dasgupta A, Kjarsgaard M, Capaldi D, et al. A pilot randomised clinical trial of mepolizumab in COPD with eosinophilic bronchitis. Eur Respir J 2017; 49: 1602486.

56 Pavord ID, Chanez P, Criner GJ, et al. Mepolizumab for eosinophilic chronic obstructive pulmonary disease. N Engl J Med 2017; 377: 1613-1629.

57 Ortega HG, Yancey SW, Mayer B, et al. Severe eosinophilic asthma treated with mepolizumab stratified by baseline eosinophil thresholds: a secondary analysis of the DREAM and MENSA studies. Lancet Respir Med 2016; 4: 549-556.

58 Harriman GR, Kunimoto DY, Elliott JF, et al. The role of IL-5 in IgA B cell differentiation. J Immunol 1988; 140: 3033-3039.

59 George L, Wright A, Mistry V, et al. Sputum Streptococcus pneumoniae is reduced in COPD following treatment with benralizumab. Int J COPD 2019; 14: 1177-1185.

60 Greulich T, Hohlfeld JM, Neuser P, et al. A GATA3-specific DNAzyme attenuates sputum eosinophilia in eosinophilic COPD patients: a feasibility randomized clinical trial. Respir Res 2018; 19: 55.

61 Snell N, Foster M, Vestbo J. Efficacy and safety of AZD1981, a CRTH2 receptor antagonist, in patients with moderate to severe COPD. Respir Med 2013; 107: 1722-1730.

62 Mahler DA, Huang S, Tabrizi M, et al. Efficacy and safety of a monoclonal antibody recognizing interleukin-8 in COPD: a pilot study. Chest 2004; 126: 926-934.

63 Kirsten AM, Forster K, Radeczky E, et al. The safety and tolerability of oral AZD5069, a selective CXCR2 antagonist, in patients with moderate-to-severe COPD. Pulm Pharmacol Ther 2015; 31: 36-41.

64 Rennard SI, Dale DC, Donohue JF, et al. CXCR2 antagonist MK-7123. A phase 2 proof-of-concept trial for chronic obstructive pulmonary disease. Am J Respir Crit Care Med 2015; 191: 1001-1011.

65 van der Vaart H, Koëter GH, Postma DS, et al. First study of infliximab treatment in patients with chronic obstructive pulmonary disease. Am J Respir Crit Care Med 2005; 172: 465-469.

66 Aaron SD, Vandemheen KL, Maltais F, et al. TNF $\alpha$ antagonists for acute exacerbations of COPD: a randomised double-blind controlled trial. Thorax 2013; 68: 142-148.

67 Rennard SI, Fogarty C, Kelsen S, et al. The safety and efficacy of infliximab in moderate to severe chronic obstructive pulmonary disease. Am J Respir Crit Care Med 2007; 175: 926-934.

68 Eich A, Urban V, Jutel M, et al. A randomized, placebo-controlled phase 2 trial of CNTO 6785 in chronic obstructive pulmonary disease. COPD 2017; 14: 476-483.

69 Calverley PMA, Sethi S, Dawson M, et al. A randomised, placebo-controlled trial of anti-interleukin-1 receptor 1 monoclonal antibody MEDI8968 in chronic obstructive pulmonary disease. Respir Res 2017; $18: 153$.

70 Novartis. Safety and Efficacy of Multiple Doses of Canakinumab (ACZ885) in Chronic Obstructive Pulmonary Disease (COPD) Patients. 2017. https://clinicaltrials.gov/ct2/show/NCT00581945?term=NCT00581945\&rank=1 Date last accessed: March 30, 2019. Date last updated: June 30, 2011.

71 Albert RK, Connett J, Bailey WC, et al. Azithromycin for prevention of exacerbations of COPD. N Engl J Med 2011; 365: 689-698.

72 Han MK, Tayob N, Murray S, et al. Predictors of chronic obstructive pulmonary disease exacerbation reduction in response to daily azithromycin therapy. Am J Respir Crit Care Med 2014; 189: 1503-1508.

73 Agustí A, Bafadhel M, Beasley R, et al. Precision medicine in airway diseases: moving to clinical practice. Eur Respir J 2017; 50: 1701655.

74 Cerquetti M, Giufrè M. Why we need a vaccine for non-typeable Haemophilus influenzae. Hum Vaccin Immunother 2016; 12: 2357-2361.

75 Lehman SM, Mearns G, Rankin D, et al. Design and preclinical development of a phage product for the treatment of antibiotic-resistant Staphylococcus aureus infections. Viruses 2019; 11: E88.

76 Lange P, Celli B, Agustí A, et al. Lung-function trajectories leading to chronic obstructive pulmonary disease. N Engl J Med 2015; 373: 111-122.

77 Postma DS, Brightling C, Baldi S, et al. Exploring the relevance and extent of small airways dysfunction in asthma (ATLANTIS): baseline data from a prospective cohort study. Lancet Respir Med 2019; 7: 402-416.

78 Soriano JB, Polverino F, Cosio BG. What is early COPD and why is it important? Eur Respir J 2018; 52: 1801448.

79 Kim V, Aaron SD. What is a COPD exacerbation? Current definitions, pitfalls, challenges and opportunites for improvement. Eur Respir J 2018; 52: 1801261.

80 de Vries R, Dagelet YWF, Spoor P, et al. Clinical and inflammatory phenotyping by breathomics in chronic airway diseases irrespective of the diagnostic label. Eur Respir J 2018; 51: 1701817.

81 Min X, Yu B, Wang F. Predictive modeling of the hospital readmission risk from patients' claims data using machine learning: a case study on COPD. Sci Rep 2019; 9: 2362.

82 Leung JM, Obeidat M, Sadatsafavi M, et al. Introduction to precision medicine in COPD. Eur Respir J 2019; 53: 1802460 